\title{
Immunization of Noncolonized Cystic Fibrosis Patients against Pseudomonas aeruginosa
}

\author{
Stanley J. Cryz, Jr., Joanna Wedgwood, Alois B. Lang, \\ Anna Ruedeberg, J. U. Que, Emil Fürer, \\ and Urs B. Schaad*
}

Swiss Serum and Vaccine Institute and Department of Pediatrics.

Inselspital. University of Berne, Berne, Switzerland

\begin{abstract}
The long-term safety and immunogenicity of a polyvalent Pseudomonas aeruginosa conjugate vaccine was evaluated in $\mathbf{3 0}$ noncolonized cystic fibrosis patients. Four doses were administered over 3 years, and patients were followed for a mean of 38 months. No acute or long-term adverse effects were noted. Immunization engendered a significant antibody response to all vaccine components. A decline in titers during year 3 of observation was associated with a marked rise in the isolation of $P$. aeruginosa. This organism was isolated repeatedly from the respiratory tract of 4 patients and only once from 7 patients. The remaining patients were repeatedly culture-negative. Only 1 patient showed clinical deterioration associated with multiple isolations of $P$. aeruginosa.
\end{abstract}

Pseudomonas aeruginosa is the leading cause of bronchopulmonary infection in patients with cystic fibrosis (CF) [1]. A substantial proportion of morbidity and mortality in this patient population is the result of recurrent pulmonary exacerbations subsequent to colonization. Rarely can $P$. aerugin$o s a$ be eradicated through antibiotic treatment. Further complicating treatment is the fact that multiply resistant strains often arise after repeated or prolonged drug therapy or prophylaxis.

Colonization of the lower respiratory tract by mucoid variants of $P$. aeruginosa is preceded by infection of the upper respiratory tract by smooth strains [2]. Such natural exposure to $P$. aeruginosa stimulates a vigorous humoral antibody response to many somatic and extracellular antigens $[3,4]$. However, such antibodies do not prevent or even ameliorate subsequent infections. In fact, several laboratories $[5,6]$ have noted a positive correlation between elevated anti- $P$. aeruginosa antibody titers and a more severe disease course. This may be the result of immune complexes being deposited in the lungs and other organs.

Anti- $P$. aeruginosa lipopolysaccharide (LPS) antibodies acquired after natural exposure are not effective at promoting opsonophagocytic killing [7]. We have found that anti$P$. aeruginosa LPS antibodies acquired subsequent to colonization had very low average affinity constants, which

Received 26 July 1993; revised 14 December 1993.

Written informed consent was obtained from patients or their guardians prior to their enrollment in this study and subsequent to the review and acceptance of the study by the Institutional Review Board of the Inselspital. Berne.

Reprints or correspondence: Dr. S. J. Cryz, Jr., Swiss Serum and Vaccine Institute, P.O. Box 2707, CH-300l Berne, Switzerland.

* Present affiliation: Department of Pediatrics, University of Basel, Basler Kinderspital, Basel, Switzerland.

The Journal of Infectious Diseases 1994;169:1159-62 (C) 1994 by The University of Chicago. All rights reserved. $0022-1899 / 94 / 6905-0040 \$ 01.00$ correlate with poor opsonic activity [8]. In contrast, vaccineinduced antibodies are opsonic and of high affinity [8].

We have recently described the safety and immunogenicity of a $P$. aeruginosa $\mathrm{O}$-polysaccharide (O-PS)-toxin A conjugate vaccine in 22 young, noncolonized $C F$ patients [9]. Vaccination resulted in a good antibody response and exerted no obvious harmful effects on clinical status. However, the comparatively short length of follow-up (mean, 18 months) did not allow firm conclusions regarding the effect of vaccination on the acquisition of $P$. aeruginosa, long-term clinical ramifications, and duration of antibody response. These studies have been expanded to include 30 noncolonized patients followed for an average of 38 months after vaccination. Various clinical, serologic, and bacteriologic parameters were analyzed.

\section{Materials and Methods}

Over 31 months, $30 \mathrm{CF}$ patients ( 13 males, 17 females) aged 1.6-24.0 years (mean, 8.3) were enrolled. All were in good health and clinically stable and had no history of infection with $P$. aeruginosa. On entry, sputum or throat swabs (or both) were negative for $P$. aeruginosa.

Patients were immunized intramuscularly at $0,2,12$, and 36 months with a low (12.5 $\mu \mathrm{g}$ of O-PS per serotype, $n=19$ ) or a high (25 $\mu \mathrm{g}$ of O-PS per serotype, $n=11$ ) dose of octavalent O-PS-toxin A conjugate vaccine [9] that contained the following International Antigen Typing System (IATS) LPS serotypes: $1,2 / 5,3,4,6,7,10$, and 16 . Patients were observed for $60 \mathrm{~min}$ after immunization. For assessment of adverse reactions, report forms were completed by the patient or parent for $96 \mathrm{~h}$ after vaccination. Each patient was examined and interviewed $\sim 1$ week after immunization. Venous blood samples were taken at the time of each immunization and at 1 - and 3-month intervals thereafter. Blood chemistry profiles were analyzed at baseline and 1 week after the first two immunizations. Disease severity, recorded at the time of the initial immunization and at 12 month intervals thereafter, was determined on the basis of sputum and throat cultures collected at 3- to 6-month intervals and 
by two scoring systems, the clinic Bernese CF score and the Chrispin-Norman radiographic score [9]. Patients were followed for a mean of 37.8 months (range, 20-46).

Total serum anti-LPS immunoglobulin $\mathrm{G}(\mathrm{IgG})$ antibody was measured by ELISA [9]. The average affinity constants for such antibodies were determined [8]. The ability of sera to neutralize the cytotoxicity of toxin A was determined in a HEp-2 cell assay [9]. $P$. aeruginosa clinical isolates were serotyped by agglutination of whole bacteria using polyclonal typing sera (Public Health Laboratory Service, London). Bronchoalveolar lavage (BAL) was done on selected patients using an Olympus (Lake Success. NY) bronchoscope. BAL fluid was plated directly onto blood agar plates, and each bacterial colony type was identified by standard laboratory techniques.

Significance of differences between antibody concentrations at various time points was assessed with a paired $t$ test.

\section{Results}

About $43 \%$ of vaccinations were associated with local reactions characterized by pain, swelling, erythema, or a combination thereof. Such reactions were predominantly mild and transient. Severity of reactions was not age-dependent and did not increase with booster doses of vaccine. There were no systemic reactions of note nor any clinically significant changes in blood chemistry parameters that could be attributed to immunization.

Because the magnitude and kinetics of the immune response did not differ substantially between the two vaccine dosages, the results were combined for ease of analysis. Representative IgG antibody responses to three LPS serotypes and to toxin $\mathrm{A}$ are shown in figure 1. The two-dose primary immunization schedule elicited a highly significant $(P<.01)$ rise in antibody titers to all nine vaccine components. Although antibody levels declined, they were still significantly $(P<.05)$ higher than baseline at 12 months. A single booster dose at this time evoked a pronounced rise in IgG antibody levels. Anti-LPS titers subsequently declined so that by 24 months, they were no longer significantly above baseline. Although antitoxin A antibody titers also fell, they remained significantly $(P<.01)$ above preimmunization levels at all time points. A second booster dose at 36 months stimulated a vigorous anti-LPS and antitoxin A antibody response with peak titers achieved 1 month later. Again, antibody levels rapidly declined over the subsequent 4 months but remained significantly $(P<.05)$ above baseline.

Before immunization, only $23 \%$ of patients possessed toxin A-neutralizing antibodies (table 1). Primary vaccination resulted in a $>10$-fold rise in mean toxin A neutralizing levels with $87 \%$ of patients seropositive. Neutralizing antibodies were maintained by most patients throughout the study with periodic boosters causing a significant $(P<.01)$ rise in neutralizing activity.

$P$. aeruginosa was never isolated from $19(61 \%)$ of the immunized patients. $P$. aeruginosa was isolated only once from

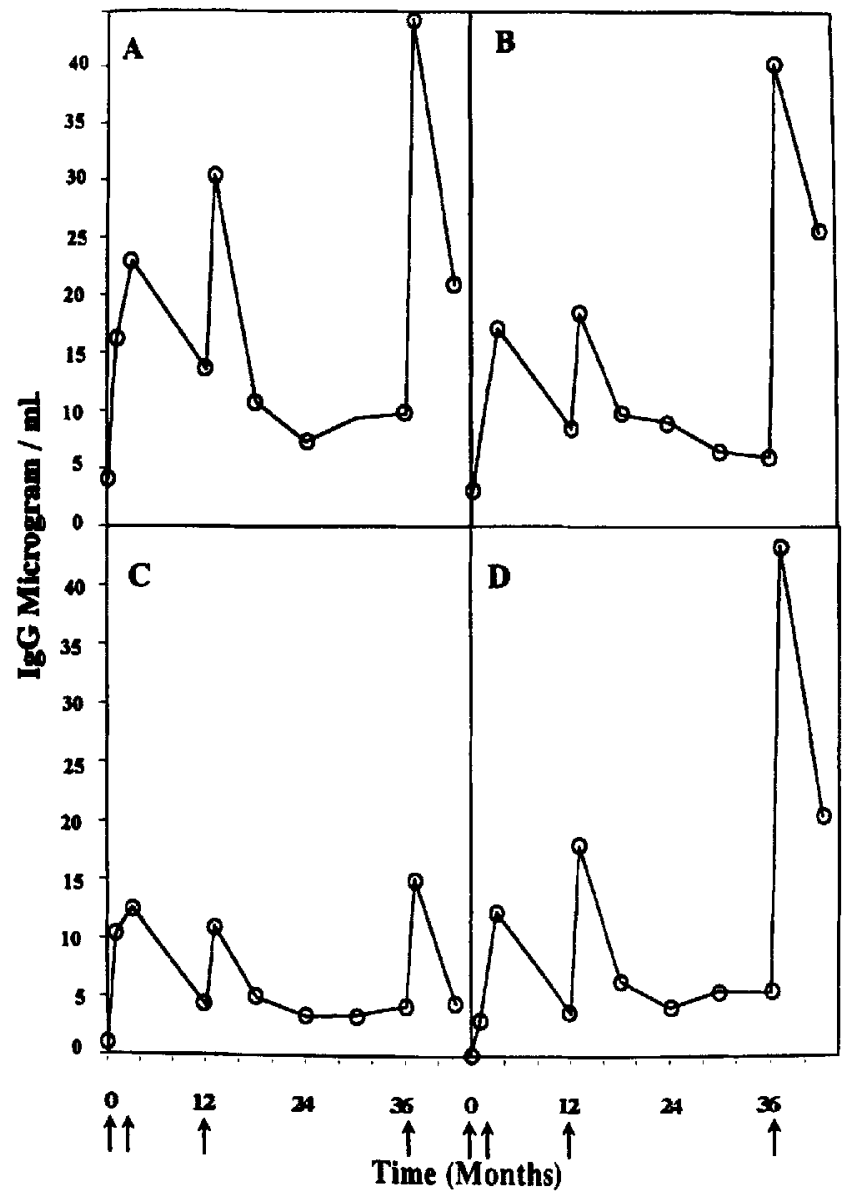

Figure 1. Lipopolysaccharide $\operatorname{IgG}$ antibody response to $\mathbf{A}$, IATS-6: B. IATS-11; C. IATS-10: and D, toxin A. Each data point represents geometric mean titer for all patients. Arrows indicate immunization times.

throat swabs or sputum cultures from 7 patients $(23 \%)$ during year 1 , from 2 during year 2 , and from 4 during year 3 . All 7 patients were culture-negative ( 6 by BAL) on subsequent culturing (6-24 months of follow-up). Four patients had $P$. aeruginosa isolated on two or more occasions. In the first patient, $P$. aeniginosa was first isolated from sputum 33 months after primary immunization. A BAL sample taken 5 months later was also positive as were 7 subsequent sputum or throat cultures taken over 10 months. This patient was hospitalized (without exacerbation) and treated with intravenous antibiotics in an attempt to eradicate $P$. aeruginosa, which was ineffective. Changes in clinical scores were associated with colonization (Chrispin-Norman score of 5 at time 0 and 7 at 36 months; Bernese score of 24 at time 0 and $2 I$ at 36 months). Significantly, only a 2- to 4-fold transient rise in anti-LPS antibody titer was engendered by vaccination.

The second patient had $P$. aeruginosa isolated (BAL and sputum) 13 months after immunization; four subsequent 
Table 1. Toxin A-neutralizing antibody engendered by immunization.

\begin{tabular}{|c|c|c|}
\hline $\begin{array}{c}\text { Time } \\
\text { (months) }\end{array}$ & $\begin{array}{c}\text { Toxin A (GM } \mu \mathrm{g}) \\
\text { neutralized*/mL of serum } \\
\text { (range) }\end{array}$ & $\begin{array}{c}\text { No. of subjects with } \\
\text { neutralizing antibody (\%) }\end{array}$ \\
\hline 0 & $<0.312(<0.312-2.5)$ & $7 / 30(23)$ \\
\hline 3 & $3.2 \quad(<0.312-20.0)$ & $26 / 30(87)$ \\
\hline 12 & $1.05 \quad(<0.312-5.0)$ & $25 / 30(83)$ \\
\hline 13 & $4.6 \quad(<0.312-20.0)$ & $29 / 30(97)$ \\
\hline 24 & $1.64(<0.312-20.0)$ & $25 / 30(83)$ \\
\hline 36 & $1.86(0.312-20.0)$ & $19 / 19(100 \%)$ \\
\hline 38 & $24.8 \quad(5-80)$ & $16 / 16(100 \%)$ \\
\hline 42 & $(1.3-20.0)$ & $9 / 9(100 \%)$ \\
\hline
\end{tabular}

NOTE. Patients were immunized at $0.2,12$, and 36 months. GM, geometric mean.

* Limit of detection was $0.312 \mu \mathrm{g}$ neutralized $/ \mathrm{mL}$ of serum.

cultures were negative. However, $P$. aeruginosa was again cultured at 37 months from BAL fluid. This patient was hospitalized (no exacerbation) after the second isolation of $P$. aeruginosa for intravenous antibiotic treatment. Clinical scores remained unchanged throughout the 42 months of follow-up. After vaccination, a good ( $\sim 10$-fold rise) antibody response was mounted and maintained to the LPS serotype expressed by the infecting strain.

$P$. aeruginosa was first isolated from sputum of the third patient 21 months after immunization, with a second isolation at 24 months. Vaccination elicited a minimal $(<2$-fold $)$ response to this serotype. Clinical scores remained essentially unchanged.

P. aeruginosa was first isolated from the fourth patient 29 months after vaccination with a second isolation at 35 months. This patient mounted an excellent immune response after vaccination and maintained titers significantly above baseline levels. $P$. aeruginosa was not isolated from this patient at a 6-month follow-up.

None of the $P$. aeruginosa strains isolated had a mucoid phenotype. Four serotypes (IATS-2/5/16, IATS-4, IATS-5, and IATS-11) were identified. One strain was nontypeable. IATS- 11 was not represented in the vaccine.

\section{Discussion}

Colonization of the lower respiratory tract of $\mathrm{CF}$ patients with $P$. aeruginosa may be viewed as a gradual, multistep process influenced by several factors, including initial colonization of the upper respiratory tract, translocation to the lower respiratory tract, decreased mucociliary clearance, concomitant infection with other bacteria or viral pathogens, $\mathrm{CF}$ transmembrane conductance regulator mutation genotype [10], and a switch from a smooth to a mucoid phenotype. Given these circumstances, preventing or even delaying the acquisition of $P$. aeruginosa could provide a substantial bene- fit to the patient. Attempts to achieve this goal with prophylactically administered antibiotics have had a modest degree of success [11].

A previous attempt to immunize noncolonized $\mathrm{CF}$ patients against $P$. aeruginos $a$ did not influence the rate of colonization [12]. Furthermore, there appeared to be a predisposition to a more severe disease course once infection occurred among the vaccinated group [12]. In contrast, we have found that multiple immunizations with a polyvalent $P$. aeruginosa vaccine over 3 years was not associated with any identifiable adverse events other than local reactions at the site of administration. The disease course did not appear to be affected by vaccination as monitored by two scoring systems nor was there any evidence of an immune complex-like syndrome. Furthermore, while 4 immunized patients appeared to become colonized or infected with $P$. aeruginosa during the course of this study, they did not undergo an unusually rapid clinical deterioration.

Immunization engendered a significant rise in $\operatorname{IgG}$ antibody levels to all vaccine constituents. Interestingly, there was a pronounced anamnestic response to booster doses administered at 12 and 36 months, a phenomenon not seen in healthy adult subjects [13]. However, there was a more rapid drop-off in antibody titers after booster doses compared with primary immunization. The kinetics and longevity of the immune response would indicate that booster vaccinations would be required at 12-to 18 -month intervals to maintain potentially protective antibody levels.

The current findings demonstrate that vaccination of noncolonized CF patients with a polyvalent O-PS-toxin A conjugate vaccine was not associated with any identifiable risks. We are now attempting to increase the immunogenicity of this vaccine with novel adjuvants. On the basis of these promising results, a double-blind placebo-controlled trial to evaluate vaccine efficacy in noncolonized CF patients may be warranted.

\section{References}

1. Marks MI. The pathogenesis and treatment of pulmonary infections in patients with cystic fibrosis. J Pediatr 1981;98:173-9.

2. Pier GB. Des Jardin D, Aquilar T. Barnard M, Speert DP. Polysaccharide surface antigens expressed by nonmucoid isolates of Psetdomonas acruginosa from cystic fibrosis patients. J Clin Microbiol 1986;24:189-96.

3. Pedersen SS. Espersen F, Høiby N. Jensen T. Immunoglobulin A and immunoglobulin $G$ antibody response to alginates from Pseudomonas aeruginosa in patients with cystic fibrosis. J Clin Microbiol 1990:28:747-55.

4. Fomsgaard A, Høiby N, Shand GH, Conrad RS, Galanos C. Longitudinal study of antibody response to lipopolysaccharides during chronic Pseudomonas aenuginosa lung infection in cystic fibrosis. Infect Immun 1988:56:2270-8.

5. Schaad UB, Lang AB, Wedgwood J, Beuhlmann U, Fürer E. Serotypespecific serum IgG antibodies to lipopolysaccharides of Pseudomonas aeruginosa in cystic fibrosis: correlation to disease, subclass distribution. and experimental protective capacity. Pediatr Res 1990;27: 508-13. 
6. van Bever HP, Gigase PL. DeClerck LS. Bridts CH, Franckx H, Stevens WJ. Immune complexes and Pseudomonas aeruginosa antibodies in cystic fibrosis. Arch Dis Child 1988;63:1222-8.

7. Eichler I, Joris L, Hsu YP, van Wye J. Bran R. Moss R. Nonopsonic antibodies in cystic fibrosis. Pseudomonas aeruginosa lipopolysaccharide-specific immunoglobulin $G$ antibodies from infected patient sera inhibit neutrophil oxidative response. J Clin Invest 1989:84: 1794-804.

8. Bruderer U, Cryz SJ Jr, Schaad UB, Deusinger M, Que JU, Lang AB. Affinity constants of naturally acquired and vaccine-induced antiPseudomonas aeruginosa antibodies in healthy adults and cystic fibrosis patients. J Infect Dis 1992;166:344-9.

9. Schaad UB, Lang AB, Wedgwood J, et al. Safety and immunogenicity of Pseudomonas ueruginosa conjugate A vaccine in cystic fibrosis. Lancet 1991:338:1236-7.
10. Kubesch P. Dörk T, Wulbrand U, et al. Genetic determinants airways' colonization with Pseudomonas aeruginosa in cystic fibiosis. Lancet 1993;341:189-93

11. Valerius NH. Koch C. Hoiby N. Prevention of chronic Pseudomonas aeruginosa colonization in cystic fibrosis by early treatment. Lancet 1991:338:725-6.

12. Longford DT. Hiller J. Prospective, controlled study of a polyvalent Pseudomonas vaccine in cystic fibrosis: three year results. Arch Dis Child 1984;59:1131-4.

13. Cryz SJ Jr. Sadoff JC. Fürer E. Immunization with a Pseudomonas aeruginosa immunotype 50 -polysaccharide-toxin A conjugate vaccine: effect of a booster dose as antibody leveis in humans. Infect Immun 1988:56:1829-30.

\title{
Mutations in the Catalase-Peroxidase Gene from Isoniazid-Resistant Mycobacterium tuberculosis Isolates
}

\author{
Manuel Altamirano, Johanna Marostenmaki, \\ Alfred Wong, Mark FitzGerald, William A. Black, \\ and John A. Smith
}

\author{
Medical Microbiology. Department of Pathology. and Division of \\ Respiratory Medicine. Department of Medicine, University of British \\ Columbia; Vancouver General Hospital; and British Columbia Centre \\ for Disease Control. Vancouver, Canada
}

\begin{abstract}
Isoniazid resistance in Mycobacterium tuberculosis has been associated with total deletion of the $k a t G$ gene, which codes for catalase-peroxidase production. To determine whether this is a common mechanism of drug resistance, 9 isolates of isoniazid-resistant and 1 of isoniazid-sensitive $M$. tuberculosis were analyzed by polymerase chain reaction amplification of a 237 -bp sequence of the $k a t G$ gene. Amplification was observed in the isoniazid-sensitive isolate and in 8 resistant isolates; in only 1 isoniazid-resistant isolate was there no amplification of the expected band, suggesting gene deletion. DNA sequencing showed that 8 of the 9 isolates had point mutations, deletions, or insertions of 1-3 bases. Evidence corroborating the presence of mutations in the kat $G$ gene was obtained by single-strand conformation polymorphism analysis in these 8 isolates. Thus, mutations as well as insertions and deletions in the $k a t G$ gene can account for inactive catalase peroxidase, leading to isoniazid resistance; gene deletion occurs only infrequently, in $\sim 11 \%$ of cases.
\end{abstract}

Tuberculosis is still a problem worldwide. It is estimated that one-third of the world's population is infected by $M y c o$ bacterium tuberculosis. Tuberculosis is among the most important infectious diseases, with 3 million deaths per year [1]. Tuberculosis is a problem not only in developing countries: Industrialized countries have experienced a decline in

Received 27 October 1993; revised 10 January 1994

Presented in part: Molecular Mechanisms of Drug Resistance, $1993 \mathrm{Al}-$ bany Conference. Albany. New York, 9-12 September 1993 (abstract 25).

Reprints or correspondence: Dr. Manuel Altamirano, Medical Microbiology. Dept. of Pathology. University of British Columbia, 2733 Heather St., Vancouver, B.C., Canada, V57. $1 \mathrm{M} 9$.

The Journal of Infectious Diseases 1994;169:1162-5 (C) 1994 by The University of Chicago. All rights reserved. $0022-1899 / 94 / 6905-0041 \$ 01.00$ tuberculosis, but since 1985 this trend has been reversed, with increases of $18.4 \%$ in the United States to $33 \%$ in Switzerland [2]. The increase in tuberculosis frequency in the United States can be correlated with the AIDS epidemic, the influx of infected immigrants, outbreaks of tuberculosis in congregative facilities [3], and numbers of homeless [4]. To complicate the problem, outbreaks of multidrug-resistant tuberculosis have been reported lately in 14 hospitals and in the prison system, with documented cases of multidrug-resistant tuberculosis in 241 patients and 15 health workers [5]. Drug resistance has been found in $60 \%$ of homeless persons with tuberculosis in Boston, $21 \%$ in New York, and 27\% in San Antonio [4]. Also, there is a rapid spread of multidrugresistant tuberculosis, especially in human immunodeficiency virus-infected patients. [6], who have a mortality rate of $\geqslant 89 \%$ [3]. This situation is reminiscent of the prechemotherapy age [7]. 